\title{
Avaliação do Perfil das Interações Medicamentosas e os Fatores Associados em Prescrições Médicas de Pacientes Internados em Unidade de Terapia Intensiva
}

\author{
Ana Paula Rodrigues Dutra '; Larissa Morgan Andrade Lemos ${ }^{2}$; Rodrigo Santos Damascena ${ }^{3}$
}

\begin{abstract}
Resumo: Em Unidades de Terapia Intensiva (UTIs), o risco de reações adversas e tratamento terapêutico ineficaz é elevado, tal fato se deve as interações medicamentosas, que podem ocorrer quando a terapêutica prescrita é composta de diversos fármacos de diferentes classes. Essas terapias com múltiplos medicamentos acontecem porque tais unidades tratam pacientes críticos. Objetivo Primário: avaliar o perfil das interações medicamentosas e os fatores associados em prescrições médicas de pacientes internados em UTI em uma unidade hospitalar de grande porte no interior da Bahia. Materiais e Métodos: Trata-se de um estudo quantitativo, descritivo, realizado em um hospital público de grande porte através da análise das prescrições médicas da Unidade de Terapia Intensiva no período de 06 de setembro a 06 de outubro de 2018. Resultados: Foram encontradas 1485 Interações Medicamentosas nas prescrições referentes a 52 pacientes que estiveram internados no período da análise documental do presente estudo. Estas foram quantificadas, tiveram sua frequência analisada e classificadas de acordo com sua relevância em Secundária, Moderada, Importante e Contraindicada. Discussão: foi identificado 63,37\% de IM-Importantes e 2,09\% das IM encontradas foram contraindicadas, tal dado é preocupante pois entre os possíveis sinais e sintomas da interação medicamentosa classificada como importante figura hipotensão, bradicardia, hipoatividade e síndromes piramidais e as IMs contraindicadas geram riscos de morte e agravos importantes a saúde do paciente. Considerações Finais: O conhecimento do perfil das IMs que ocorrem em um determinado contexto assistencial contribui para a elaboração de ações de prevenção e o acompanhamento dos resultados clínicos das interações pela equipe de saúde
\end{abstract}

Palavras-chave: Interação Medicamentosa, Unidade de Terapia Intensiva, Prescrições.

\section{Evaluation of the Profile of Medicinal Interactions and the Factors Associated with Medical Prescriptions of Injured Patients in an Intensive Therapy Unit}

\begin{abstract}
In Intensive Care Units (ICUs), the risk of adverse reactions and ineffective therapeutic treatment is high, due to drug interactions, which may occur when the prescribed therapy is composed of several drugs of different classes, these multi-drug therapies they happen because such units treat critical patients. Primary Objective: to evaluate the profile of drug interactions and associated factors in medical prescriptions of ICU patients in a large hospital unit in the interior of Bahia. Materials and Methods: This is a quantitative, descriptive study carried out in a large public hospital through the analysis of the medical prescriptions of the Intensive Care Unit from September 6 to October 6, 2018. Results: found 1485 Drug Interactions in the prescriptions related to 52 patients who were hospitalized in the period of the documentary analysis of the present study, these were
\end{abstract}

\footnotetext{
${ }^{1}$ Graduanda em Farmácia pela Faculdade Independente do Nordeste - FAINOR. Vitória da Conquista/BA. Brasil. Endereço: Av. São Luiz, N. 31, Bairro Candeias, CEP: 45.055-080, Vitória da Conquista - BA. E-mail: dutraa.anapaula@ gmail.com

2 Mestre em Saúde Coletiva/UFBA. Especialista em Assistência Farmacêutica/UEFS. Docente Adjunta do Curso de Farmácia/FAINOR. Vitória da Conquista/BA, Brasil. Endereço: Av. São Luiz, N. 31, Bairro Candeias, CEP: 45.055-080, Vitória da Conquista - BA. E-mail: larissamorgan@hotmail.com

${ }^{3}$ Graduação em Ciências Farmacêuticas pela Universidade Estadual de Feira de Santana. Mestre em Saúde Pública pela Fiocruz. Especialista em Microbiologia Clínica. Especialista em Saúde Pública com Ênfase em PSF. Especialista em Gestão da Assistência Farmacêutica. Especialista em Farmácia Clínica e Hospitalar. Vitória da Conquista - BA. E-mail: rodrigodamascena12@gmail.com>
} 
quantified, had their frequency analyzed and classified according to their relevance in Secondary, Moderate, Important and Contraindicated. Discussion: $63.37 \%$ of IM-Importants and $2.09 \%$ of IMs were found to be contraindicated. This is a cause for concern because among the possible signs and symptoms of drug interaction, hypotension, bradycardia, hypoactivity and pyramidal syndromes the contraindicated IMs generate risks of death and important health problems to the patient. Final considerations: The knowledge of the profile of IMs that occur in a particular care context contributes to the elaboration of prevention actions and the monitoring of the clinical results of the interactions by the health team

Keywords: Drug Interaction, Intensive Care Unit, Prescriptions.

\section{Introdução}

O risco de interações medicamentosas está intimamente relacionado com o uso simultâneo de mais de um medicamento, que é algo muito comum na terapêutica hospitalar. Justifica-se a utilização de vários medicamentos quando existe a necessidade de uma ação sinérgica no tratamento medicamentoso, ou para a terapia de várias doenças simultâneas, no entanto pode contribuir para uma interação entre os fármacos. Esta conduta é chamada de polifármacia, sendo muito comum no ambiente hospitalar e ao mesmo tempo é particularmente crítica, pois durante o período de internamento, os pacientes fazem uso de vários medicamentos diferentes (MOURA; RIBEIRO; MAGALHÃES, 2007).

Quando a interação medicamentosa diminui os efeitos indesejados e aumenta a eficácia do tratamento ou reduzem seus efeitos adversos são classificadas como benéficas, já quando leva a uma potencialização do efeito, reduzindo a eficácia terapêutica, causando toxicidade ou reações adversas, sendo ela grave ou não, são classificados como prejudiciais (RODRIGUES, PINHO, 2013).

Geralmente em Unidades de Terapia Intensiva (UTIs), o risco de reações adversas e tratamento terapêutico ineficaz é elevado. Tal fato se deve as interações medicamentosas, que podem ocorrer quando a terapêutica prescrita é composta de diversos fármacos de diferentes classes. Essas terapias com múltiplos medicamentos acontecem porque tais unidades tratam pacientes críticos, dentre esses destaca-se a presença de idosos e portadores de polipatologias (VIEIRA et al., 2012).

Sabendo que existe a possibilidade de IM, é necessário atentar-se para elementos decisivos que contribuem para sua ocorrência, tais como a natureza química do fármaco, quantidade de medicamentos utilizados, presença de pacientes com idade avançada, hepatopatas, portadores de problemas respiratórios, cardiomiopatias, hipotireoidismo, epilepsia grave e nefropatas. Esses últimos, por serem portadores de Doenças Renais Crônicas (DRC), 
compõem uma população de alto risco para desenvolvimento de IM graves, pois há um predomínio de idosos, hipertensos e diabéticos na composição dessa classe, condição que é agravada por existir uma dificuldade de excreção renal de medicamentos (PAULA et al., 2015).

Outro fator que determina se a interação medicamentosa vai se tornar um Evento Clínico Adverso (ECA) é a significância clínica de uma interação. Além dos fatores relacionados aos pacientes, as IM estão associadas a fatores referente às propriedades farmacológicas dos medicamentos a que os pacientes estão sendo submetidos no decorrer da terapia medicamentosa prescrita (PAULA et al., 2015).

De acordo com Passos (2012), quando se trata dos fármacos, se refere a capacidade do mesmo em induzir e inibir enzimas e a margem terapêutica específica de cada fármaco, que são os aspectos mais relevantes, com relação a prescrição médica. A periodicidade de IM é mínima em pacientes em uso de um número inferior a 4 medicamentos e aumenta em pacientes que faz uso de 10 a 20 medicamentos, ou seja, a prevalência no número de IM durante o internamento dentre a população hospitalizada varia de 37 a $80 \%$, a depender do volume de fármacos e tempo de permanência na UTI.

O potencial das interações medicamentosa são avaliadas em geral pelas pesquisas através de prontuário ou prescrições médicas apesar de ainda a gravidade e incidência de algumas interações ainda não serem conhecidas, de acordo com a pesquisa realizada por Moura, Ribeiro e Magalhães (2014) a incidência das IMs a nível hospitalar varia entre 5,4\% e 69,7\%.

Este estudo possui como objetivo avaliar o perfil das interações medicamentosas e os fatores associados em prescrições médicas de pacientes internados em UTI em uma unidade hospitalar de grande porte no interior da Bahia.

A realização desse estudo é justificado devido ao alto risco que as IMs podem trazer aos pacientes hospitalizados que se encontram em tratamento medicamentoso, auxiliando assim na escolha da terapêutica que mais se adequada a cada quadro clínico, além desse fato é de suma importância realizar estudos que possam contribuir para a disseminação desse assunto em toda comunidade científica para garantir que o paciente tenha a intervenção terapêutica, com o devido controle dos riscos em que ele estará sujeito.

\section{Materiais e Métodos}

Trata-se de um estudo quantitativo, descritivo, realizado em um hospital público de grande porte, situado no interior da Bahia. Este é administrado por gestão dupla direta, com 
oferta de atenção nos níveis de média e alta complexidade, possui pactuação com 72 municípios, o atendimento hospitalar é ininterrupto, incluindo plantões aos finais de semana e feriados (BAHIA, 2018). A unidade conta atualmente com 40 leitos de UTI destinados a adultos, distribuídos em 3 serviços, dois desses serviços contam com 10 leitos cada disponíveis para atendimento ao público e o terceiro serviço possui 20 leitos.

O estudo foi realizado através da análise das prescrições médicas dos setores 1 e 2, no período de 06 de setembro a 06 de outubro de 2018, o terceiro serviço não compôs o local de estudo, pois a mesma foi inaugurado no segundo semestre de 2018 e esta não estava em funcionamento na época que o projeto de pesquisa que embasou esse estudo foi confeccionado.

Os documentos utilizados para a captação de dados, foram todas as prescrições impressas das Unidades de Terapia Intensiva em estudo. Esta unidade como muitos no Brasil, não possui prescrição eletrônica, desta forma, diariamente, a prescrição é elaborada pelo médico prescritor e nela consta toda terapêutica necessários para as próximas 24 horas de internamento na UTI.

O critério de inclusão utilizado para captar os sujeitos da pesquisa era o paciente ser submetidos a tratamento medicamentoso com dois ou mais fármacos e que apresentassem permanência mínima de uma diária na UTI. Utilizou-se como critérios excludentes pacientes com idade inferior a 18 anos e/ou que permanecessem no internamento por menos de um dia. Ao aplicar tais critérios no período estudado foram analisadas as prescrições diárias de 52 pacientes enquanto estes permaneceram internados no setor estudado.

Foram analisados nas prescrições das Unidades de Terapia Intensiva, sexo, idade e medicamentos prescritos. O estudo analisou as interações fármaco-fármaco, os fármacos e as interações mais corriqueiras após análise foram relatadas e submetidas à classificação de Interações Medicamentosas Potenciais Teóricas (IMPT).

As IM encontradas foram analisadas com base nas informações do banco de dados Micromedex®, assim foi possível levantar dados acerca das implicações a saúde dos pacientes ou reações adversas que decorrem a partir da IM e classifica-las de acordo com a gravidade. Salienta-se que o Ácido Acetilsalicílico, Clorpromazina, Fenitoína, Cetoprofeno, Cefalotina, Etilefrina não foram incluídos no estudo, pois o Micromedex ${ }^{\circledR}$ não engloba tais fármacos em sua base de dados.

A quantificação dos dados foi executada com auxílio do software Microsoft Excel® (versão 2013) no qual foi possível realizar uma análise estatística dos dados encontrados e assim criar um perfil das IMs e a frequência que a mesma teve no período do estudo, o perfil da idade e sexo dos sujeitos da pesquisa. Estes dados foram organizadas sob a forma de tabelas, pois tal 
recurso permite que exista uma visualização facilitada dos dados encontrados e foi utilizado software Microsoft Word® (versão 2013) para o processamento de texto.

A pesquisa foi aprovada junto ao Comitê de Ética em Pesquisa com Seres Humanos da Faculdade Independente do Nordeste (Fainor) sob o parecer 2.883.665.

\section{Resultados}

Foram analisados os prontuários de 52 pacientes que estiveram em Internamento nas Unidades de Terapia Intensiva em estudo, durante os 30 dias em que a pesquisa aconteceu. A Tabela 1 traz a distribuição dos pacientes de acordo o sexo e idade, sendo 26 (50\%) eram do sexo Masculino e 26 (50\%) do sexo Feminino. A Idade Média foi de 57,53 \pm 19,19 anos, sendo que dos 52 participantes, 5 não tiveram a idade coletada por falta de informações no prontuário.

Tabela 1 - Distribuição dos pacientes de acordo o sexo e idade

\begin{tabular}{ccccc}
\hline \multirow{2}{*}{ IDADE } & \multicolumn{2}{c}{ Feminino } & \multicolumn{2}{c}{ Mexo } \\
& $\mathrm{n}$ & $\%$ & $\mathrm{~N}$ & $\%$ \\
\hline $20-29$ & 1 & $2 \%$ & 2 & $4 \%$ \\
$30-39$ & 5 & $10 \%$ & 4 & $8 \%$ \\
$40-49$ & 3 & $6 \%$ & 2 & $4 \%$ \\
$50-59$ & 2 & $4 \%$ & 3 & $6 \%$ \\
$60-69$ & 3 & $6 \%$ & 8 & $15 \%$ \\
$70-79$ & 3 & $6 \%$ & 4 & $8 \%$ \\
$>90$ & 4 & $8 \%$ & 3 & $6 \%$ \\
Não informado & 5 & $10 \%$ & - & $0 \%$ \\
Total & $\mathbf{2 6}$ & $\mathbf{5 0 \%}$ & $\mathbf{2 6}$ & $\mathbf{5 0 \%}$ \\
\hline
\end{tabular}

Fonte: Dados da Pesquisa. Vitória da Conquista. 2018.

Foram encontradas 1485 Interações Medicamentosas no período de análise documental do estudo, estas foram classificadas de acordo com sua relevância em Secundária, Moderada, Importante e Contraindicada. A Tabela 2 dispõe a contagem das IMs pela relevância sendo um dado alarmante que 63,37\% (941 Interações), foram de Importante Relevância e 2,09\% (31 interações) eram contraindicadas. 
Tabela 2 - Distribuição das Interações Medicamentosas de acordo a Relevância

\begin{tabular}{ccc}
\hline Classificação & Número de Interação Medicamentosa & Frequência \\
\hline CONTRAINDICADO & 31 & $2,09 \%$ \\
IMPORTANTE & 941 & $63,37 \%$ \\
MODERADA & 499 & $33,60 \%$ \\
SECUNDARIA & 14 & $0,94 \%$ \\
Total Geral & 1485 & $100,00 \%$
\end{tabular}

Fonte: Dados da Pesquisa. Vitória da Conquista. 2018.

A Tabela 3 traz a lista de 183 Interações Medicamentosas diferentes encontradas durante a coleta de dados, em ordem decrescente da frequência com que foram encontradas.

Tabela 3 - Lista das Interações Medicamentosas Encontradas, Número e Frequência de ocorrências e sua relevância.

\begin{tabular}{|c|c|c|c|}
\hline Interação Medicamentosa & Número & Frequência & Relevância \\
\hline MIDAZOLAM X OMEPRAZOL & 98 & $6,82 \%$ & MODERADA \\
\hline FENTANIL X MIDAZOLAM & 96 & $6,68 \%$ & IMPORTANTE \\
\hline DIPIRONA X ENOXAPARINA & 78 & $5,42 \%$ & IMPORTANTE \\
\hline FENTANIL X METOCLOPRAMIDA & 34 & $2,36 \%$ & IMPORTANTE \\
\hline INSULINA NPH X METOCLOPRAMIDA & 34 & $2,36 \%$ & IMPORTANTE \\
\hline DIPIRONA X FUROSEMIDA & 33 & $2,29 \%$ & IMPORTANTE \\
\hline DIPIRONA X LOSARTANA & 32 & $2,23 \%$ & MODERADA \\
\hline FENTANIL X FLUCONAZOL & 27 & $1,88 \%$ & IMPORTANTE \\
\hline DIPIRONA X HEPARINA & 26 & $1,81 \%$ & IMPORTANTE \\
\hline INSULINA REGULAR X METOCLOPRAMIDA & 26 & $1,81 \%$ & IMPORTANTE \\
\hline METOCLOPRAMIDA X MORFINA & 24 & $1,67 \%$ & IMPORTANTE \\
\hline BACLOFENO X FENTANIL & 23 & $1,60 \%$ & IMPORTANTE \\
\hline CLOPIDOGREL X ENOXAPARINA & 23 & $1,60 \%$ & IMPORTANTE \\
\hline CLOPIDOGREL X OMEPRAZOL & 23 & $1,60 \%$ & IMPORTANTE \\
\hline FLUCONAZOL X LOSARTANA & 22 & $1,53 \%$ & MODERADA \\
\hline FUROSEMIDA X INSULINA NPH & 22 & $1,53 \%$ & MODERADA \\
\hline AMIODARONA X METADONA & 19 & $1,32 \%$ & IMPORTANTE \\
\hline METADONA X METOCLOPRAMIDA & 19 & $1,32 \%$ & IMPORTANTE \\
\hline CLOPIDOGREL X SINVASTATINA & 18 & $1,25 \%$ & MODERADA \\
\hline METOCLOPRAMIDA X MIDAZOLAM & 18 & $1,25 \%$ & IMPORTANTE \\
\hline FUROSEMIDA X INSULINA REGULAR & 17 & $1,18 \%$ & MODERADA \\
\hline FUROSEMIDA X MORFINA & 17 & $1,18 \%$ & MODERADA \\
\hline HIDROCLOROTIAZIDA X INSULINA NPH & 16 & $1,11 \%$ & MODERADA \\
\hline FLUCONAZOL X MIDAZOLAM & 15 & $1,04 \%$ & MODERADA \\
\hline INSULINA REGULAR X TIMOLOL & 15 & $1,04 \%$ & MODERADA \\
\hline INSULINA NPH X TIMOLOL & 14 & $0,97 \%$ & MODERADA \\
\hline
\end{tabular}




\begin{tabular}{|c|c|c|c|}
\hline DEXAMETASONA X FENTANIL & 13 & $0,90 \%$ & IMPORTANTE \\
\hline DIPIRONA X HIDROCLOROTIAZIDA & 13 & $0,90 \%$ & IMPORTANTE \\
\hline FLUCONAZOL X METRONIDAZOL & 13 & $0,90 \%$ & IMPORTANTE \\
\hline FENTANIL X METADONA & 12 & $0,83 \%$ & IMPORTANTE \\
\hline ISONIAZIDA X PREDNISONA & 12 & $0,83 \%$ & SECUNDARIA \\
\hline AMITRIPTILINA X DIPIRONA & 11 & $0,76 \%$ & IMPORTANTE \\
\hline DIPIRONA X METOPROLOL & 11 & $0,76 \%$ & MODERADA \\
\hline FUROSEMIDA X PANCURONIO & 11 & $0,76 \%$ & MODERADA \\
\hline DIPIRONA X PREDNISONA & 10 & $0,70 \%$ & IMPORTANTE \\
\hline FLUCONAZOL X METADONA & 10 & $0,70 \%$ & CONTRAINDICADO \\
\hline HEPARINA X PAROXETINA & 10 & $0,70 \%$ & IMPORTANTE \\
\hline METOCLOPRAMIDA X TRAMADOL & 10 & $0,70 \%$ & IMPORTANTE \\
\hline PAROXETINA X TRAMADOL & 10 & $0,70 \%$ & IMPORTANTE \\
\hline AMIODARONA X FENTANIL & 9 & $0,63 \%$ & IMPORTANTE \\
\hline AMIODARONA X FLUCONAZOL & 9 & $0,63 \%$ & CONTRAINDICADO \\
\hline BACLOFENO X METADONA & 9 & $0,63 \%$ & IMPORTANTE \\
\hline FLUCONAZOL X TRAMADOL & 9 & $0,63 \%$ & IMPORTANTE \\
\hline BACLOFENO X METOCLOPRAMIDA & 8 & $0,56 \%$ & IMPORTANTE \\
\hline CLOPIDOGREL X DIPIRONA & 8 & $0,56 \%$ & IMPORTANTE \\
\hline FENTANIL X KETAMINA & 8 & $0,56 \%$ & IMPORTANTE \\
\hline LORAZEMPAM X METADONA & 8 & $0,56 \%$ & IMPORTANTE \\
\hline LORAZEPAM X METOCLOPRAMIDA & 8 & $0,56 \%$ & IMPORTANTE \\
\hline OMEPRAZOL X RIFAMPICINA & 8 & $0,56 \%$ & MODERADA \\
\hline ONDANSETRONA X TRAMADOL & 8 & $0,56 \%$ & IMPORTANTE \\
\hline PIRAZINAMIDA X RIFAMPICINA & 8 & $0,56 \%$ & IMPORTANTE \\
\hline AMITRIPTILINA X FLUCONAZOL & 7 & $0,49 \%$ & IMPORTANTE \\
\hline AMITRIPTILINA X METOCLOPRAMIDA & 7 & $0,49 \%$ & CONTRAINDICADO \\
\hline AMITRIPTILINA X MORFINA & 7 & $0,49 \%$ & IMPORTANTE \\
\hline CIPROFLOXACINO X METRONIDAZOL & 7 & $0,49 \%$ & IMPORTANTE \\
\hline FENTANIL X PAROXETINA & 7 & $0,49 \%$ & IMPORTANTE \\
\hline FENTANIL X RIFAMPICINA & 7 & $0,49 \%$ & IMPORTANTE \\
\hline GABAPENTINA X MORFINA & 7 & $0,49 \%$ & MODERADA \\
\hline INSULINA REGULAR X LOSARTANA & 7 & $0,49 \%$ & MODERADA \\
\hline AMITRIPTILINA X TRAMADOL & 6 & $0,42 \%$ & IMPORTANTE \\
\hline CARVEDILOL X DIPIRONA & 6 & $0,42 \%$ & MODERADA \\
\hline DIAZEPAM X OMEPRAZOL & 6 & $0,42 \%$ & SECUNDARIA \\
\hline FLUCONAZOL X RISPERIDONA & 6 & $0,42 \%$ & IMPORTANTE \\
\hline HIDROCLOROTIAZIDA X INSULINA REGULAR & 6 & $0,42 \%$ & MODERADA \\
\hline INSULINA NPH X LOSARTANA & 6 & $0,42 \%$ & MODERADA \\
\hline METADONA X METRONIDAZOL & 6 & $0,42 \%$ & IMPORTANTE \\
\hline METADONA X MIDAZOLAM & 6 & $0,42 \%$ & IMPORTANTE \\
\hline MIDAZOLAM X RIFAMPICINA & 6 & $0,42 \%$ & MODERADA \\
\hline
\end{tabular}




\begin{tabular}{|c|c|c|c|}
\hline OMEPRAZOL X PROPRANOLOL & 6 & $0,42 \%$ & MODERADA \\
\hline AMIODARONA X CIPROFLOXACINO & 5 & $0,35 \%$ & IMPORTANTE \\
\hline AMIODARONA X METRONIDAZOL & 5 & $0,35 \%$ & IMPORTANTE \\
\hline AMIODARONA X TRAMADOL & 5 & $0,35 \%$ & IMPORTANTE \\
\hline CLINDAMICINA X PANCURÔNIO & 5 & $0,35 \%$ & MODERADA \\
\hline CLONAZEPAM X TRAMADOL & 5 & $0,35 \%$ & IMPORTANTE \\
\hline CLONIDINA X INSULINA REGULAR & 5 & $0,35 \%$ & MODERADA \\
\hline DEXAMETASONA X TRAMADOL & 5 & $0,35 \%$ & IMPORTANTE \\
\hline DIPIRONA X TIMOLOL & 5 & $0,35 \%$ & MODERADA \\
\hline ENOXAPARINA X HEPARINA & 5 & $0,35 \%$ & IMPORTANTE \\
\hline ENOXAPARINA X PAROXETINA & 5 & $0,35 \%$ & IMPORTANTE \\
\hline ENOXAPARINA X RIVAROXABANA & 5 & $0,35 \%$ & IMPORTANTE \\
\hline FENTANIL X HALOPERIDOL & 5 & $0,35 \%$ & IMPORTANTE \\
\hline FENTANIL X ONDANSETRONA & 5 & $0,35 \%$ & IMPORTANTE \\
\hline FENTANIL X TRAMADOL & 5 & $0,35 \%$ & IMPORTANTE \\
\hline FLUCONZAOL X OMEPRAZOL & 5 & $0,35 \%$ & MODERADA \\
\hline METADONA X RANITIDINA & 5 & $0,35 \%$ & IMPORTANTE \\
\hline METADONA X RISPERIDONA & 5 & $0,35 \%$ & IMPORTANTE \\
\hline METADONA X TRAMADOL & 5 & $0,35 \%$ & IMPORTANTE \\
\hline MIDAZOLAM X TRAMADOL & 5 & $0,35 \%$ & IMPORTANTE \\
\hline MORFINA X ONDANSETRONA & 5 & $0,35 \%$ & IMPORTANTE \\
\hline AMIODARONA X LOSARTANA & 4 & $0,28 \%$ & MODERADA \\
\hline ATENOLOL X DIPIRONA & 4 & $0,28 \%$ & MODERADA \\
\hline ATRACURIO X CLINDAMICINA & 4 & $0,28 \%$ & MODERADA \\
\hline CIPROFLOXACINO X TRAMADOL & 4 & $0,28 \%$ & IMPORTANTE \\
\hline CLONIDINA X INSULINA NPH & 4 & $0,28 \%$ & MODERADA \\
\hline CLOPIDOGREL X LANSOPRAZOL & 4 & $0,28 \%$ & IMPORTANTE \\
\hline DEXAMETASONA X DIPIRONA & 4 & $0,28 \%$ & IMPORTANTE \\
\hline DIAZEPAM X METOCLOPRAMIDA & 4 & $0,28 \%$ & IMPORTANTE \\
\hline ESPIRONOLACTONA X LOSARTANA & 4 & $0,28 \%$ & MODERADA \\
\hline FENTANIL X LORAZEPAM & 4 & $0,28 \%$ & IMPORTANTE \\
\hline FETANIL X METOCLOPRAMIDA & 4 & $0,28 \%$ & IMPORTANTE \\
\hline HIDROCLOROTIAZIDA X PREDNISONA & 4 & $0,28 \%$ & MODERADA \\
\hline KETAMINA X METOCLOPRAMIDA & 4 & $0,28 \%$ & IMPORTANTE \\
\hline LANSOPRAZOL X PROPRANOLOL & 4 & $0,28 \%$ & MODERADA \\
\hline METADONA X PREDNISONA & 4 & $0,28 \%$ & IMPORTANTE \\
\hline PREDNISONA X RIFAMPICINA & 4 & $0,28 \%$ & MODERADA \\
\hline AC. VALPROICO X ERTAPENEM & 3 & $0,21 \%$ & IMPORTANTE \\
\hline AMIODARONA X ANLODIPINO & 3 & $0,21 \%$ & IMPORTANTE \\
\hline CARVEDILOL X DOBUTAMINA & 3 & $0,21 \%$ & MODERADA \\
\hline CIPROFIBRATO X SINVASTATINA & 3 & $0,21 \%$ & IMPORTANTE \\
\hline CLONIDINA X HALOPERIDOL & 3 & $0,21 \%$ & MODERADA \\
\hline
\end{tabular}




\begin{tabular}{|c|c|c|c|}
\hline CLONIDINA X RISPERIDONA & 3 & $0,21 \%$ & MODERADA \\
\hline DIAZEPAM X MORFINA & 3 & $0,21 \%$ & IMPORTANTE \\
\hline DIPIRONA X PROPRANOLOL & 3 & $0,21 \%$ & MODERADA \\
\hline DIPIRONA X RIVAROXABANA & 3 & $0,21 \%$ & IMPORTANTE \\
\hline FENTANIL X PREDNISONA & 3 & $0,21 \%$ & IMPORTANTE \\
\hline HALOPERIDOL X PROMETAZINA & 3 & $0,21 \%$ & IMPORTANTE \\
\hline HALOPERIDOL X TRAMADOL & 3 & $0,21 \%$ & IMPORTANTE \\
\hline AC. VALPROICO X LORAZEPAM & 2 & $0,14 \%$ & MODERADA \\
\hline AMIODARONA X ATENOLOL & 2 & $0,14 \%$ & MODERADA \\
\hline AMIODARONA X CARVEDILOL & 2 & $0,14 \%$ & MODERADA \\
\hline AMIODARONA X CLONAZEPAM & 2 & $0,14 \%$ & MODERADA \\
\hline AMIODARONA X SINVASTATINA & 2 & $0,14 \%$ & IMPORTANTE \\
\hline AMITRIPTILINA X DIAZEPAM & 2 & $0,14 \%$ & MODERADA \\
\hline AMITRIPTILINA X FENTANIL & 2 & $0,14 \%$ & IMPORTANTE \\
\hline CIPROFLOXACINO X FENTANIL & 2 & $0,14 \%$ & IMPORTANTE \\
\hline CLOPIDOGREL X HEPARINA & 2 & $0,14 \%$ & MODERADA \\
\hline DEXAMETASONA X PANCURONIO & 2 & $0,14 \%$ & MODERADA \\
\hline DIAZEPAM X FENTANIL & 2 & $0,14 \%$ & IMPORTANTE \\
\hline DIPIRONA X ENALAPRIL & 2 & $0,14 \%$ & MODERADA \\
\hline FENTANIL X QUETIAPINA & 2 & $0,14 \%$ & IMPORTANTE \\
\hline FENTANIL X RANITIDINA & 2 & $0,14 \%$ & IMPORTANTE \\
\hline FLUCONAZOL X ONDANSETRONA & 2 & $0,14 \%$ & CONTRAINDICADO \\
\hline FUROSEMIDA X HIDROCORTISONA & 2 & $0,14 \%$ & MODERADA \\
\hline FUROSEMIDA X PROPRANOL & 2 & $0,14 \%$ & MODERADA \\
\hline HALOPERIDOL X RISPERIDONA & 2 & $0,14 \%$ & IMPORTANTE \\
\hline LEVOTIROXINA X OMEPRAZOL & 2 & $0,14 \%$ & MODERADA \\
\hline LORAZEPAM X MORFINA & 2 & $0,14 \%$ & IMPORTANTE \\
\hline METRONIDAZOL X ONDANSETRONA & 2 & $0,14 \%$ & IMPORTANTE \\
\hline QUETIAPINA X RISPERIDONA & 2 & $0,14 \%$ & IMPORTANTE \\
\hline AMIODARONA X METOPROLOL & 1 & $0,07 \%$ & MODERADA \\
\hline AMIODARONA X MORFINA & 1 & $0,07 \%$ & IMPORTANTE \\
\hline AMIODARONA X ONDANSETRONA & 1 & $0,07 \%$ & IMPORTANTE \\
\hline AMIODARONA X RANITIDINA & 1 & $0,07 \%$ & IMPORTANTE \\
\hline ANLODIPINO X SINVASTATINA & 1 & $0,07 \%$ & IMPORTANTE \\
\hline ATENOLOL X CLONIDINA & 1 & $0,07 \%$ & MODERADA \\
\hline ATRACURIO X HIDROCORTIZONA & 1 & $0,07 \%$ & MODERADA \\
\hline BACLOFENO X TRAMADOL & 1 & $0,07 \%$ & IMPORTANTE \\
\hline CARVEDILOL X INSULINA REGULAR & 1 & $0,07 \%$ & MODERADA \\
\hline CARVEDILOL X MORFINA & 1 & $0,07 \%$ & IMPORTANTE \\
\hline CIPROFLOXACINO X HIDROCORTISONA & 1 & $0,07 \%$ & IMPORTANTE \\
\hline CIPROFLOXACINO X DEXAMETASONA & 1 & $0,07 \%$ & IMPORTANTE \\
\hline CIPROFLOXACINO X METOPROLOL & 1 & $0,07 \%$ & SECUNDARIA \\
\hline
\end{tabular}




\begin{tabular}{|c|c|c|c|}
\hline CLONAZEPAM X METOCLOPRAMIDA & 1 & $0,07 \%$ & IMPORTANTE \\
\hline CLONAZEPAM X MORFINA & 1 & $0,07 \%$ & IMPORTANTE \\
\hline CLOPIDOGREL X FENTANIL & 1 & $0,07 \%$ & IMPORTANTE \\
\hline CLOPIDOGREL X MORFINA & 1 & $0,07 \%$ & IMPORTANTE \\
\hline DIPIRONA X LEVOFLOXACINO & 1 & $0,07 \%$ & MODERADA \\
\hline FENTANIL X MORFINA & 1 & $0,07 \%$ & IMPORTANTE \\
\hline FLUCONAZOL X HALOPERIDOL & 1 & $0,07 \%$ & CONTRAINDICADO \\
\hline FLUCONAZOL X QUETIAPINA & 1 & $0,07 \%$ & IMPORTANTE \\
\hline HALOPERIDOL X MOTOCLOPRAMIDA & 1 & $0,07 \%$ & CONTRAINDICADO \\
\hline HALOPERIDOL X ONDANSETRONA & 1 & $0,07 \%$ & IMPORTANTE \\
\hline HALOPERIDOL X QUETIAPINA & 1 & $0,07 \%$ & IMPORTANTE \\
\hline HEPARINA X NITROGLICERINA & 1 & $0,07 \%$ & IMPORTANTE \\
\hline HIDROCLOROTIAZIDA X MORFINA & 1 & $0,07 \%$ & MODERADA \\
\hline INSULINA REGULAR X LEVOFLOXACINO & 1 & $0,07 \%$ & IMPORTANTE \\
\hline LEVOFLOXACINO X PREDNISONA & 1 & $0,07 \%$ & IMPORTANTE \\
\hline CLOPIDROGREL X MORFINA & 1 & $0,07 \%$ & IMPORTANTE \\
\hline METADONA X MORFINA & 1 & $0,07 \%$ & IMPORTANTE \\
\hline METADONA X ONDANSENTRONA & 1 & $0,07 \%$ & IMPORTANTE \\
\hline METADONA X QUETIAPINA & 1 & $0,07 \%$ & IMPORTANTE \\
\hline METOCLOPRAMIDA X RISPERIDONA & 1 & $0,07 \%$ & CONTRAINDICADO \\
\hline MIDAZOLAM X RANITIDINA & 1 & $0,07 \%$ & MODERADA \\
\hline MORFINA X METOCLOPRAMIDA & 1 & $0,07 \%$ & IMPORTANTE \\
\hline MORFINA X PROMETAZINA & 1 & $0,07 \%$ & IMPORTANTE \\
\hline MORFINA X TRAMADOL & 1 & $0,07 \%$ & IMPORTANTE \\
\hline PIPERACILINA / TAZOBACTAM X VANCOMICINA & 1 & $0,07 \%$ & IMPORTANTE \\
\hline PREDNISONA X PROPRANOLOL & 1 & $0,07 \%$ & MODERADA \\
\hline PROPRANOLOL X OMEPRAZOL & 1 & $0,07 \%$ & MODERADA \\
\hline PROPRANOLOL X RIFAMPICINA & 1 & $0,07 \%$ & MODERADA \\
\hline RANITIDINA X RISPERIDONA & 1 & $0,07 \%$ & MODERADA \\
\hline ISONIAZIDA X RIFAMPICINA & 0 & $0,00 \%$ & IMPORTANTE \\
\hline
\end{tabular}

Fonte: Dados da Pesquisa. Vitória da Conquista. 2018.

\section{Discussão}

Reis et al., (2016) caracterizou em seu estudo, os pacientes de uma Unidade de Terapia Intensiva Adulta com características similares a da unidade em estudo e os autores trouxeram em sua pesquisa que idades variavam entre 18 e 86 anos apresentando uma idade média de 46,4 anos. Esse dado destoa da idade média da população em estudo que aparecem com uma idade média de 57,53 anos (Tabela 1). 
No entanto, no estudo de Moura, Ribeiro e Magalhães, (2007) os autores trazem que não foi possível verificar uma associação entre idade e a quantidade de medicamentos prescritos, ou ainda entre idade e potencial de ocorrência de interações medicamentosas. Segundo os autores, isso pode ter ocorrido, em parte, pelo fato de o material de análise ter sido a prescrição e não o paciente, desta forma, como foi analisada somente uma prescrição por paciente, pode ter desconsiderado outros medicamentos prescritos.

De acordo com Ciampone et al., (2006), um dos fatores que devem ser ponderados é o quesito idade, pois levanta questões acerca do emprego de recursos na Unidades de Terapia Intensiva e a ponderação entre o custo e o benefício nesses setores de alta complexidade, pelo fato da população está envelhecendo, aumenta-se a expectativa de vida e junto se aumenta a frequência de patologias com características de cronicidade e degeneração do Sistema Nervoso Central (SNC). Estas geram agravos à saúde e consequentemente, a necessidade de intervenções complexas que exigem internação em UTI.

Nascimento et al. (2016) realizou um estudo em um hospital geral de grande porte do estado da Paraíba, onde foram analisados 215 prontuários referentes aos internamentos da Unidade de Terapia Intensiva, e ao traçar o perfil epidemiológico da amostra, os autores evidenciaram uma prevalência do sexo masculino de 54\% dos internamentos. Tais dados são similares aos encontrados no estudo onde traz uma prevalência de $50 \%$ dos internamentos serem por homens (Tabela 1).

Segundo Cedraz, (2014) é essencial destacar a prevalência de IM classificadas como moderadas e importantes. Tal fato mostra a relevância clínica que deve ser dada às IM classificadas como moderadas, uma vez que o programa Thomsom Micromedex as caracteriza como interações que podem provocar uma exacerbação da condição do paciente e/ou necessitar de uma mudança na terapia medicamentosa.

Em um estudo realizado por Alvim et al., (2015) em uma Unidade de Terapia Intensiva em um hospital de ensino do estado de Minas Gerais, ao analisar as prescrições eletrônicas dos pacientes que foram internados nessa UTI durante o ano de 2015, constatou uma frequência que destoa da encontrada no estudo (Tabela 2), pois 41,84\% das IM foram classificadas como Importantes e 9,18\% como Contraindicadas. O presente estudo revelou IM-Importantes em $63,37 \%$ e 2,09\% das IM encontradas foram contraindicadas. Tal dado é preocupante pois entre os possíveis sinais e sintomas da interação medicamentosa classificada como importante figura hipotensão, bradicardia, hipoatividade e síndromes piramidais e as IMs contraindicadas geram riscos de morte e agravos importantes a saúde do paciente. As Interações Medicamentosas Contraindicadas (Tabela 3) encontradas durante a análise documental das prescrições médicas 
foram: FLUCONAZOL $X$ METADONA, AMIODARONA X FLUCONAZOL, AMITRIPTILINA $\mathrm{X}$ METOCLOPRAMIDA, FLUCONAZOL $\mathrm{X}$ ONDANSETRONA, FLUCONAZOL X HALOPERIDOL, HALOPERIDOL X MOTOCLOPRAMIDA $\mathrm{e}$ METOCLOPRAMIDA X RISPERIDONA.

Existe a interação medicamentosa aplicada com finalidade terapêutica, a mais utilizada foi entre midazolam e fentanil. Foi comparada que é eficaz associar tais medicamentos para sedar os pacientes em ventilação mecânica invasiva, do que o uso apenas do midazolam, em um teste clínico feito de forma aleatório e cega. Foi observado que a administração simultânea desses fármacos com auxílio de Bomba de Infusão Contínua (BIC) leva os pacientes a uma sedação facilitada e utilizando dosagens menores, ao comparar com o uso de apenas midazolam.

O quantitativo de eventos adversos se mantém nos mesmos níveis, é interessante destacar que na interação midazolam x fentanil, foi constatado determinadas reações adversas a exemplo de hipotensão, diminuição da frequência respiratória e a consequente queda da saturação de oxigênio, o que justifica a classificação dessa interação como Importante (GIMENES; BARONI; RODRIGUES, 2014). O estudo revelou que foi realizado 96 vezes (Tabela 3) essa interação nos dois serviços de Unidade de Terapia Intensiva em estudo, sendo a segunda maior frequência de IM do estudo, 6,68\%, dado esse que nos mostra que é corriqueira esse tipo de interação medicamentosa tendo por finalidade auxiliar na sedação do paciente.

Cedraz, (2014) mostra em seu estudo a presença de interações que deveriam ser ao máximo evitadas como por exemplo o simultâneo de Metoclopramida e antipsicóticos (Haloperidol) que pode elevar as chances de reações extrapiramidais ou síndrome maligna dos neurolépticos, por isso é caracterizada como contraindicada, no estudo tal reação teve $0,07 \%$ de frequência (Tabela 3).

Mazzola, (2011) chamam a atenção em seu estudo para a necessidade de atenção com medicamentos que são utilizados frequentemente na prática clínica, mas que requerem atenção e acompanhamento de seu uso, quando estão associados com outras classes de fármacos. Um exemplo é a metoclopramida, que quando associados com agentes neurolépticos é contra indicado devidos os seus riscos, apesar que a incidência dos efeitos da interação não sejam muito conhecidas. A metoclopramida está presente neste estudo em 7 das interações medicamentosas potenciais teóricas identificadas como contraindicadas.

Destaca-se a amiodarona, como um dos medicamentos mais relacionados em ocasionar interações medicamentosas. Pelo fato deste fármaco possuir meia vida longa e variável, a chance de acontecer interações medicamentosas é relevante, considerando que a amiodarona é um substrato da CYP3A4 e todos os outros medicamentos que tem essa características do 
processo de ligação, inibição ou indução da CYP3A4 correm o risco de interações medicamentosas, sendo fundamental uma atenção criteriosa nestes casos (GIMENES; BARONI; RODRIGUES, 2014). No estudo em questão não foi diferente, mostrando diversas interações envolvendo a amiodarona, dentre elas está Amiodarona X Fluconazol, com frequência de $0,63 \%$ no presente estudo é classificada como contraindicada, devido aos seus riscos de cardiotoxicidade.

Na pesquisa realizada por Passos, (2012) observou-se um predomínio de medicamentos que pertence às seguintes classes terapêuticas: Anticoagulantes, Anti-Hipertensivos e Analgésicos. As associações de fármacos mais constantes nas IM foram a Dipirona que quando interage com medicamentos do sistema cardiovascular leva a uma redução do efeito hipertensivo e natriunrético, da mesma maneira que a Heparina eleva o risco de hemorragia, assim como na caracterização dos medicamentos e das IM deste estudo.

Em um estudo realizado por Mazzola, (2011), entre as interações identificadas como maiores, destacou-se a prevalência da interação entre dipirona e a enoxaparina, pelo fato de tal interação como indicação de manejo clínico, ser necessário a suspensão da dipirona quando possível, ou que seja mantida por meio de acompanhamento contínuo de prováveis riscos de sangramento. Mesmo com tais recomendações, sabe-se pouco a respeito da incidência desta interação e desta forma é fundamental uma avaliação criteriosa de cada paciente para determinar a relação risco benefício de uma suspensão ou de uma alteração da terapia medicamentosa. Durante as análises para essa pesquisa tal interação apareceu 78 vezes, caracterizando uma frequência de 5,42\% das interações medicamentosas importantes.

As interações medicamentosas recorrentes na unidade, sendo elas moderadas ou graves, podem ser controladas não apenas pela suspensão da associação terapêutica, mas por ajuste de doses e acompanhamento de possíveis efeitos adversos, realizando uma avaliação específica de risco e benefício (YUNES, COELHO, ALMEIDA, 2011).

\section{Considerações Finais}

É possível na prescrição, dispensação e administração dos medicamentos compreender a natureza, o risco e a gravidade potencial de se associar um medicamento a outro, a identificação das interações medicamentosas permite evitar situações de falha terapêutica e/ou diminuir o aparecimento de toxicidade medicamentosa tanto pelo ajuste do esquema posológico como pelo uso de fármacos alternativos. 
Um dos fatores que mais afeta o tratamento são IM, e o significado clínico é de difícil prognostico. Além da análise das prescrições investigando-se a presença de IM e a realização de recomendações, o farmacêutico clínico tem várias atuações referentes à terapia farmacológica nos serviços de saúde, no qual deve influenciar na segurança do paciente.

O conhecimento do perfil das IMs que ocorrem em um determinado contexto assistencial contribui para a elaboração de ações de prevenção e o acompanhamento dos resultados clínicos das interações pela equipe de saúde, dessa forma, a gravidade, o índice terapêutico e classe farmacológica dos medicamentos envolvidos, constitui os problemas clínicos para o paciente e a característica das evidências científicas disponíveis indica a significância clínica de uma IM, fazendo com que seja imprescindíveis que exista a detecção, uma análise dos documentos e viabilização de soluções dos problemas oriundos do uso incorreto dos fármacos.

\section{Referências}

ALVIM, M.M. et al. Eventos adversos por interações medicamentosas potenciais em unidade de terapia intensiva de um hospital de ensino. Rev Bras Ter Intensiva. 2015.

BRASIL. Conselho Nacional de Saúde. Resolução n 466, de 12 de dezembro de 2012. Aprova normas regulamentadoras de pesquisas envolvendo seres humanos. Brasília: Diário Oficial da União, 2013.

CEDRAZ, S. J. M. C. D. Identificação e caracterização de interações medicamentosas em prescrições médicas da unidade de terapia intensiva de um hospital público da cidade de Feira de Santana , BA. Rev Soc Bras Clin Med, v. 55, n. 75, 2014.

CIAMPONE ET AL., 2005. Necessidades de cuidados de enfermagem e intervenções terapêuticas em Unidade de Terapia Intensiva : estudo comparativo entre pacientes idosos e não idosos Nursing care need and therapeutics interventions in Intensive Care Unit: de Cuidad. v. 19, n. 1, p. 28-35, 2016.

FONSECA, J. J. S. et al. Metodologia do Trabalho Científico. São Carlos: Serviço de Biblioteca e Informação, 2014.

FONTANA, V. B. Estudo da frequencia de interação medicamentosa em prescrições médicas contendo medicamentos de baixo índice terapêutico, (Monografia - Univates). 2013.

GERHARDT, T.E., SILVEIRA,D.T. A pesquisa científica. Porto Alegre: Editora da UFRGS, 2015 
GIMENES, BARONI, RODRIGUES, 2014. Interações MedIcamentosas Potenciais em Unidade de Terapia Intensiva Adulto de um Hospital Publico Estadual. Rev. Bras. Farm. Hosp. Serv. Saúde São Paulo, v. 5 n.4, p. 19-24, 2014.

MOURA, C. S.; RIBEIRO, A. Q.; MAGALHÃES, S. M. S. Avaliação de interações medicamentosas potenciais em prescrições médicas do Hospital das Clínicas da Universidade Federal de Minas Gerais (Brasil). Latin American Journal of Pharmacy, v. 26, n. 4, p. 596$601,2007$.

NASCIMENTO, M.S.M. et al. PERFIL EPIDEMIOLÓGICO DE PACIENTES EM UNIDADE DE TERAPIA INTENSIVA ADULTO DE UM HOSPITAL REGIONAL PARAIBANO. Temas em Saúde. Volume 18, N 1, João Pessoa-PB, 2018.

NOGUEIRA, L. DE S. et al. Características clínicas e gravidade de pacientes internados em UTIS públicas e privadas. Texto e Contexto Enfermagem, v. 21, n. 1, p. 59-67, 2012.

NOVARETTI, M. C. Z. et al. Sobrecarga de trabalho da Enfermagem e incidentes e eventos adversos em pacientes internados em UTI. Revista Brasileira de Enfermagem, v. 67, n. 5, p. 692-699, out. 2014.

NOVARETTI ET AL., 2014. Nursing workload and occurrence of incidents and adverse events in ICU patients. Revista Brasileira de Enfermagem, v. 67, n. 5, p. 692-699, 2014.

OLIVEIRA, L. P.; ZAGO, K. S. DE A.; AGUIAR, S. B. Potenciais interações medicamentosas em um serviço de urgência psiquiátrica de um hospital geral: análise das primeiras vinte e quatro horas. SMAD. Revista Eletrônica Saúde Mental Álcool e Drogas (Edição em Português), v. 11, n. 4, p. 190, 2015.

PASSOS, M. et al. Interações medicamentosas em pacientes internados na clínica médica de um hospital de ensino e fatores Associados. v. 93, n. 4, p. 450-456, 2012.

PAULA, V. C. DE et al. Avaliação de eventos clínicos adversos decorrentes de interações medicamentosas em uma unidade de terapia intensiva de um hospital universitário Assessment of adverse clinical events resulting from drug interactions in an intensive care unit of a universi.

Boletim informativo Geum, v.6,n.3,p.83-90,jul./set.2015, p. 83-90, 2015.

PIOVESAN, A., TEMPORINI, E.R. Pesquisa exploratória: procedimento metodológico para o estudo de fatores humanos no campo da saúde pública. Revista de Saúde Pública, 29 (4) 318$25,2015$.

PRAÇA, F. S. G. METODOLOGIA DA PESQUISA CIENTÍFICA: ORGANIZAÇÃO ESTRUTURAL E OS DESAFIOS PARA REDIGIR O TRABALHO DE CONCLUSÃO Revista Eletrônica "Diálogos Acadêmicos" (ISSN: 0486-6266).

REIS, L.C.C. et al. As repercussões do processo de internação em UTI adulto na perspectiva de familiares. Temas psicol. vol.24 no.3 Ribeirão Preto set. 2016

VIEIRA, L. B. et al. Interações medicamentosas potenciais em pacientes de Unidades de Terapia Intensiva TT - Potential drug interactions in Intensive Care Unit patients. Rev. ciênc. 
farm. básica apl, v. 33, n. 3, p. 401-408, 2012.

YUNES, COELHO, A. 2011. Principais interações medicamentosas em pacientes da Uti-adulto de um hospital privado de Minas Gerais. R. Bras. Farm. Hosp. Serv. Saúde São Paulo, v. 2 n.3, n. 61, p. 23-26, 2011.

\section{Como citar este artigo (Formato ABNT):}

DUTRA, Ana Paula Rodrigues; LEMOS, Larissa Morgan Andrade; DAMASCENA, Rodrigo Santos. Avaliação do Perfil das Interações Medicamentosas e os Fatores Associados em Prescrições Médicas de Pacientes Internados em Unidade de Terapia Intensiva. Id on Line Rev.Mult. Psic., 2019, vol.13, n.43, p. 543-558. ISSN: 1981-1179.

Recebido: 22/11/2018;

Aceito: 23/11/2018 